\title{
Bit error rate performance on passive alignment in free space optical links using large core fibers
}

Bryan Schoenholz, Sarah Tedder, Patrick Millican, Joel Berkson

Bryan Schoenholz, Sarah Tedder, Patrick Millican, Joel Berkson, "Bit error rate performance on passive alignment in free space optical links using large core fibers," Proc. SPIE 10524, Free-Space Laser Communication and Atmospheric Propagation XXX, 1052415 (15 February 2018); doi: $10.1117 / 12.2290201$

SPIE. Event: SPIE LASE, 2018, San Francisco, California, United States 


\title{
Bit error rate performance on passive alignment in free space optical links using large core fibers
}

\author{
Bryan Schoenholz*a, Sarah Tedder ${ }^{\mathrm{a}}$, Patrick Millican ${ }^{\mathrm{b}}$, Joel Berkson ${ }^{\mathrm{c}}$ \\ ${ }^{a}$ NASA Glenn Research Center, 21000 Brookpark Rd, Cleveland, OH, USA 44135; 'bniversity of \\ Notre Dame Department of Physics, 225 Nieuwland Science Hall, Notre Dame, IN USA 46556; \\ 'University of Arizona College of Optical Sciences, 1630 E. University Blvd, Tucson, AZ USA
}

85721

\begin{abstract}
A 20-meter free-space optical link (FSOL) is proposed for data transmission between external ISS payload sites and the main cabin at a target rate of $10 \mathrm{Gbps}$ (gigabits per second). Motion between a payload site and the main cabin is predicted to cause up to $5 \mathrm{~cm}$ in lateral misalignment and 0.2 degrees of angular misalignment. Due to the harsh environment of space it is advantageous to locate the optical transceivers inside the spacecraft or in a controlled environment. With the optical components and transceivers in separate locations, a fiber optic cable will be required to carry light between the two. In our past work we found that the use of large-core fibers provide an increased misalignment tolerance for such systems and could eliminate the need for active control of the optics. In that work, it was shown that a $105 \mu \mathrm{m}$ core diameter fiber optic cable offered a viable low SWaP (size, weight, and power) solution for the ISS application; however, the effects of modal dispersion were not investigated. This paper will present bit error rate performance of the FSOL using these large-core fibers.
\end{abstract}

Keywords: Free Space Optical Link, Optical Bit Error Rate Testing, Modal Dispersion in Large Core Fibers

\section{INTRODUCTION}

Free space optical links (FSOL) can enable extremely high rate wireless communication systems. One application that NASA is interested in using FSOL's is to transmit large quantities of data between payload sites on the International Space Station (ISS). An intra ISS application will pose many challenges which include the harsh space environment and dynamic misalignment between nodes. Due to extreme temperature swings and exposure to radiation it is advantageous to develop a system that is capable of locating the optical transceiver and its associated electronics within the spacecraft while keeping transmit and receive optical assemblies outside. This enables better control of the environment with which these very sensitive transceiver components are housed. In this system architecture a fiber optic cable is needed to carry light between the optical transceivers and the transmit/receive optical assemblies. The motion between a typical external payload site on the ISS and the main cabin is predicted to cause up to $5 \mathrm{~cm}$ in lateral misalignment and 0.2 degrees of angular misalignment of the optics ${ }^{1}$. In order to develop a low size weight and power (SWaP) solution, passive misalignment mitigation solutions are being investigated. In our past work ${ }^{2}$ we presented data from a 20 meter FSOL which emulated a potential link between the ExPRESS (expedite the processing of experiments to the Space Station) Logistics Carrier (ELC) and the main cabin. The results of that work show that a passive system at $1 \mathrm{Gbps}$ is capable of tolerating the ISS motion by leveraging two key components: an optimized divergence of the transmitted beam and the use of a $105 \mu \mathrm{m}$ core multi-mode fiber (MMF) optic cable to increase the receiver's field of view. In that study, received power was the only figure of merit to determine the systems potential lateral misalignment tolerance. This paper will experimentally investigate the bit error rate (BER) performance of this system at the higher rate of $10 \mathrm{Gbps}$ in the presence of lateral misalignment. Two areas of BER degradation are expected: signal to noise ratio (SNR) degradation due to misalignment of the optical sub-assemblies and modal dispersion in the large core fiber.

*bryan.schoenholz-1@ @asa.gov; phone 1216 433-8053; nasa.gov

Free-Space Laser Communication and Atmospheric Propagation XXX, edited by Hamid Hemmati,

Don M. Boroson, Proc. of SPIE Vol 10524, 1052415 - (c) 2018 SPIE

CCC code: $0277-786 \mathrm{X} / 18 / \$ 18 \cdot$ doi: $10.1117 / 12.2290201$

Proc. of SPIE Vol. 10524 1052415-1 


\section{MEASURMENT SYSTEM}

The BER measurements taken for this study fall within in two main configurations: a fiber only system and a free space system. First, a fiber only system is tested to isolate the effects of modal dispersion in the MMF cable and show its relationship to BER performance. Then a free space system is tested to investigate the effects of lateral misalignment on BER performance. The system parameters which are varied are: the length of the MMF cable used at the receive optic, the divergence of the transmitted beam in free space, and the amount of lateral misalignment between the transmit and receive optics. This will allow us to investigate how the cable length of the MMF at the receiver affects system performance. Equations $1-3^{3}$ are used to approximate the bit rate capacity of the MMF cables to be tested.

$$
\begin{aligned}
& \tau_{i} \approx \frac{L}{2 n_{1} c}(N A)^{2} \\
& \tau_{m}=D_{m} \times L \times \Delta \lambda \\
& B_{\text {max }}=\frac{0.7}{\sqrt{\tau_{i}^{2}+\tau_{m}^{2}}}
\end{aligned}
$$

Where, $L$ is the length of the cable under test in meters, $n_{l}$ is the core index of refraction at $1550 \mathrm{~nm}, N A$ is numerical aperture, $c$ is the speed of light, $D_{m}$ is the material dispersion coefficient at $1550 \mathrm{~nm}, \Delta \lambda$ is the spectral width, and $B_{\max }$ is the maximum data rate supported by the cable due to modal dispersion. Figure 1 shows the relationship between the MMF length and its theoretical maximum bit rate for the test fibers used in this experiment.

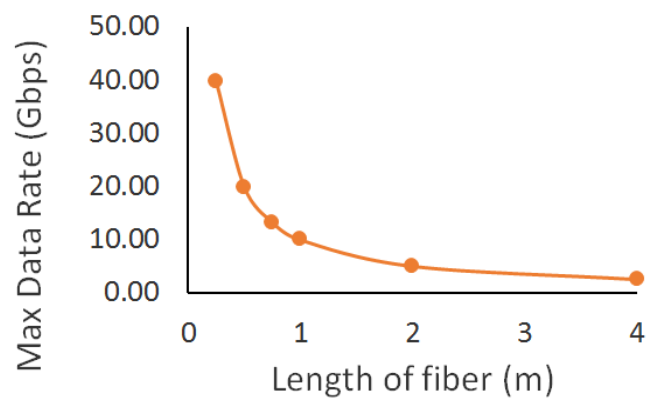

Figure 1. Theoretical Maximum Data Rate vs. Fiber Length for MMF with $n_{1}=1.4439$, NA $=0.22, D_{m}=21.5187 \mathrm{ps} / \mathrm{nm}$ $\mathrm{km}, \Delta \lambda=2 \mathrm{~nm}$, and $c=3 \mathrm{e} 8 \mathrm{~m} / \mathrm{s}$

Using the results of Figure 1 the cable lengths chosen for this study are 1 meter, 2 meters and 4 meters for both the fiber only test configuration and the free space test configuration.

\subsection{Fiber only System}

The fiber only measurement system uses an MP2100A Antrsu BertWave instrument to send out a $2^{31}$ pseudo random binary sequence (PRBS) at 10 Gbps over low voltage differential signals (LVDS). The signal is then sent to an extended reach (ZR) small form factor pluggable optical transceiver (SFP+) specified for $10 \mathrm{Gbps}$ over $80 \mathrm{~km}$ of single mode fiber and has a quoted receiver sensitivity of $-24 \mathrm{dBm}$. The SFP+ is installed on an SFP+ evaluation board to allow direct access to device bias voltage and all input/output signals. The SFP+ modulates $1550 \mathrm{~nm}$ light using on-off keying (OOK) and sends it into a single mode fiber (SMF). The light is then attenuated using a digital step attenuator and sent to a 50/50 optical splitter. Half of the light is then measured using a fiber coupled power meter (PM) while the rest is launched into a MMF which serves as the device under test (DUT). The light is launched into the MMF using a simple FC/FC (ferrule connector) mating sleeve so that the face of each cable mates and aligns their optical axis together. The MMF's used are step index cables with a $105 \mu \mathrm{m}$ pure silica core and 125 um fluorine-doped silica cladding. The 105 $\mu \mathrm{m}$ core was chosen as it is the largest core size available that we could get fabricated with an LC (Lucent) connector mated on one end for interfacing with the SFP+ and an FC/UPC (Ferrule Connector/Ultra Physical Contact) connector on the other end to interface with the optical sub-assemblies. This MMF/DUT carries light back to the SFP+ which converts the received light back to LVDS pulses which are analyzed by the BertWave. The SFP+ also reports its received optical power level over the Digital Diagnostic Monitoring Interface (DDMI) using an I2C bus that is received 
by an external I2C host adaptor. Custom Python control software running on a windows computer is used as a master controller and interfaces with the host adaptor, step attenuator, and BertWave to log all data and status during the measurement.

\subsection{FSOL System}

The measurement system shown in Figure 2 was used to quantify the BER performance of a 20 meter FSOL. Just like the fiber based system the FSOL system uses an Antrsu BertWave, SFP+, SFP+ evaluation board, I2C host adaptor and control software. In this test however the step attenuator is removed and the light is launched to free space and propagates 20 meters. This is done using a 0.25 numerical aperture (NA) aspheric lens with a focal length of $11 \mathrm{~mm}$ at an adjustable distance from the end of a SMF. Control of this distance allows adjustments to the transmitted beams divergence angle. 20 meters from the transmitter, at the receiving end of the system, a second 0.25 NA lens with $11 \mathrm{~mm}$ focal length is used to collect light and focus it onto the same multi-mode fiber (MMF) as used with the fiber only system. Again this $105 \mu \mathrm{m}$ MMF serves as the device under test (DUT). The system is then tested at various levels of lateral misalignment of the optics by using a vertical motor as shown in Figure 2.

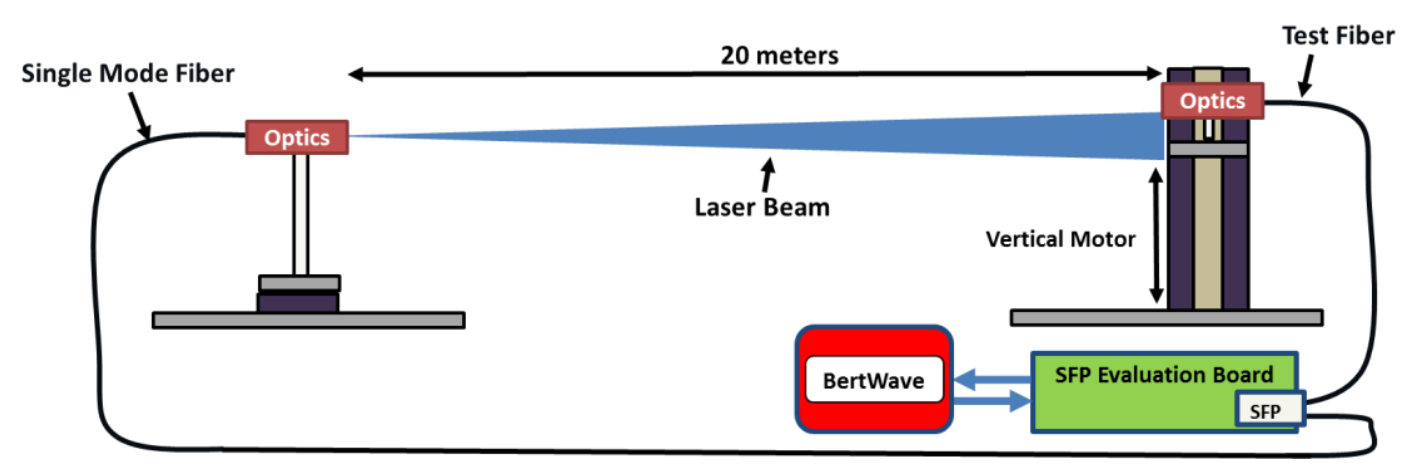

Figure 2. Free space BER measurement system block diagram.

\subsection{System Automation}

The automation of collecting BER data, received power levels, and stimulating the system all with a single control system allows for quick repeatable measurements. In both the fiber and free space system the BER measurement points are run and periodically monitored until a user defined confidence level $\left(\mathrm{C}_{\mathrm{L}}\right)$ is reached for the measurement. A Poisson distribution is used to determine the confidence level of the BER measurement. Equation 4 is used to determine $\mathrm{C}_{\mathrm{L}}$ in the case where no errors occur during the measurement ${ }^{4}$.

$$
\mathrm{C}_{\mathrm{L}}=1-e^{-N_{\text {biss }} * B E R}
$$

Where $\mathrm{C}_{\mathrm{L}}$ is confidence level (with no errors in the measurement), $\mathrm{N}_{\mathrm{bits}}$ is the number of bits evaluated, and BER is the reported bit error rate. At $10 \mathrm{Gbps}$ a 10 second measurement time is typical so that in the $\mathrm{N}_{\text {bits }}=100$ billion bits and we can be $99 \%$ confident that the BER is 1e-10 or lower. Equation 5 is used to find the confidence level with errors detected within the measurement ${ }^{4}\left(\mathrm{C}_{\mathrm{LE}}\right)$.

$$
\mathrm{C}_{\mathrm{LE}}=1-\left(e^{-N_{\text {bis }} * B E R} * \frac{1}{N_{\text {error }} !} *\left(B E R * N_{\text {bits }} N_{\text {error }}\right)\right) .
$$

Where $\mathrm{N}_{\text {error }}$ is the number of errors reported in the measurement. When the desired $\mathrm{C}_{\mathrm{L}}$ or $\mathrm{C}_{\mathrm{LE}}$ is reached the control software then stimulates the system into the next measurement point and this process is repeated until the link is lost. The $\mathrm{C}_{\mathrm{L}}$ and $\mathrm{C}_{\mathrm{LE}}$ were set to $95 \%$ or higher for measurements presented in this paper. 


\section{BER DEGREDATION SOURCES}

Two main sources of BER performance degradation can be expected when using MMF's in an optical communications system: SNR degradation and modal dispersion. SNR degradation is expected in this test system as the signal power is decreased due to coupling losses from optical misalignment. As the transmit and receive optics are laterally misaligned the signal power decreases, however the noise level remains constant causing lower SNR. Also, modal dispersion is expected to occur as light propagates through the MMF. Various spatial modes within the MMF can be excited causing changes in the pulses received at the detector.

\subsection{Measured SNR Degradation}

The relationship between received power at the SFP+ and lateral misalignment for both a collimated beam and divergent beam in free space are shown in Figure 3. This data can also be used in later sections to relate misalignment to BER. In taking this data we found that the average coupling losses between large MMF and SFP+ to be $10.4 \mathrm{~dB}$ with a standard deviation of $0.8 \mathrm{~dB}$. The low coupling efficiency into the SFP+ can be explained by the MMF overfilling a 50 micron fiber stub used in the SFP+'s Receiver Optical Sub-Assembly (ROSA), which is designed to interface with a SMF. In other words the MMF launches light outside the small core of the ROSA and much of the power is lost at the interface.

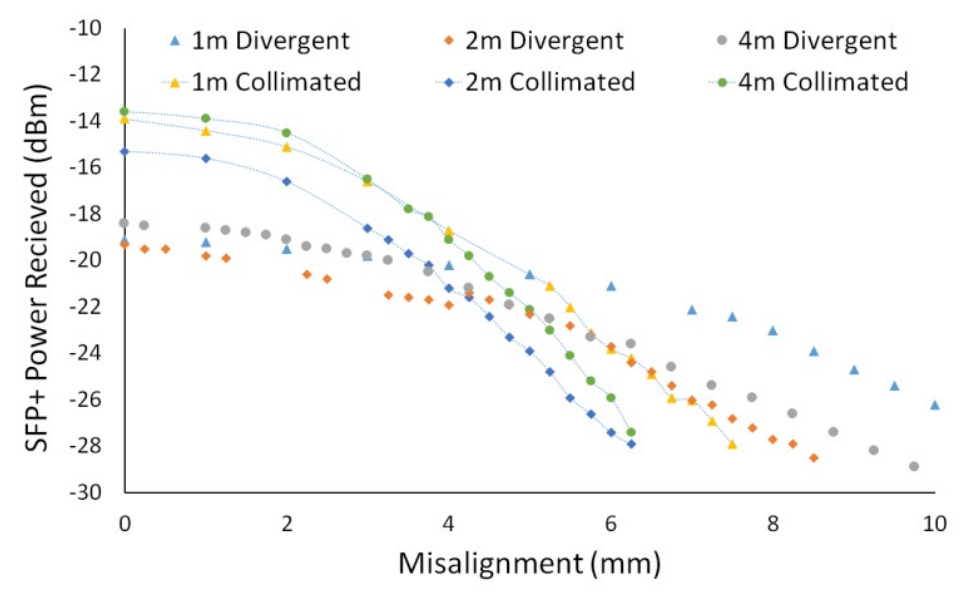

Figure 3. Received SFP+ power over lateral misalignment.

With the signal power at the detector understood in the presence of misalignment, the system noise was then investigated to fully understand the SNR of the received signal. A tungsten light was used to create white noise at a higher power density then the observed ambient lighting. This noise was added into the system through a fiber combiner to ensure it was well coupled into the system. This noise was added after a signal attenuator which was used to dial in specific signal power levels. This setup allowed for independent control of the signal power and noise power. The combined signal was then sent to an optical spectrum analyzer to measure both the signal level and in band noise power. The noise power was measured at $-78.1 \mathrm{dBm} / \mathrm{nm}$ with the tungsten light off and $-61.8 \mathrm{dBm} / \mathrm{nm}$ with the tungsten light on. BER measurements were then taken for different signal power levels both with the light on and off. The results showed equal performance between added optical noise with the light on and lower optical noise with the light off. So for our test setup the SNR is driven primarily by the received power levels of the transmitted signal through the optical system, this is because the electrical noise in the system is greater than that of optical noise coupled in to the detector.

\subsection{Modal Dispersion Theory}

The large core fiber needed to increase the receiver field of view comes at the cost of modal dispersion. Modal dispersion occurs when the fiber optic cable supports the propagation of multiple spatial modes having different group velocities and 
therefore different group delays ${ }^{5}$. As the different modes travel at different group velocities they will arrive at the detector at different times causing signal pulse broadening. This pulse broadening then leads to intersymbol interference and inherently degrades the BER performance of the system. The equation for pulse broadening is shown below in Equation $6^{6}$.

$$
\Delta \tau=L\left(\frac{1}{\left(v_{g}\right)_{l_{\max }, m_{\max }}}-\frac{1}{\left(v_{g}\right)_{0,0}}\right)
$$

Where $\Delta \tau$ is the pulse broadening, $\mathrm{L}$ is the cable length, and $\mathrm{v}_{\mathrm{g}}$ is group velocity for modes from $(0,0)$ to $\left(\mathrm{l}_{\max }, \mathrm{m}_{\max }\right)$ or the highest mode exited. This formula shows that the cable length is directly proportional to pulse broadening.

\section{RESULTS}

Various data sets were taken to show trends in the performance between two key system parameters: beam divergence and MMF cable length. Figures 4 a) and b) show how cable length affects the system performance of the 20 meter FSOL. Figure 4 a) compares the different cable lengths using a collimated beam while Figure 4 b) uses a divergent beam. As expected from Equation 6, the 1 meter cable (triangles) which is the shortest length causes the least pulse broadening and performs the best. The error rate then increases as the cable length increases for a given signal power. The trend of decreased performance as the cable length increases is observed for both the collimated beam and divergent beam. By plotting BER against received power the effect of SNR between the various cable lengths can be removed by looking at common power levels. Common power levels imply common SNR points (as described in the section above). For example, in Figure 4 a), at a received power level of $-22 \mathrm{dBm}$ the error rate is approximately $5 \mathrm{e}-7$ for the 1 meter cable, $7 \mathrm{e}-5$ for the 2 meter cable and $3 \mathrm{e}-4$ for the 4 meter cable.

a)

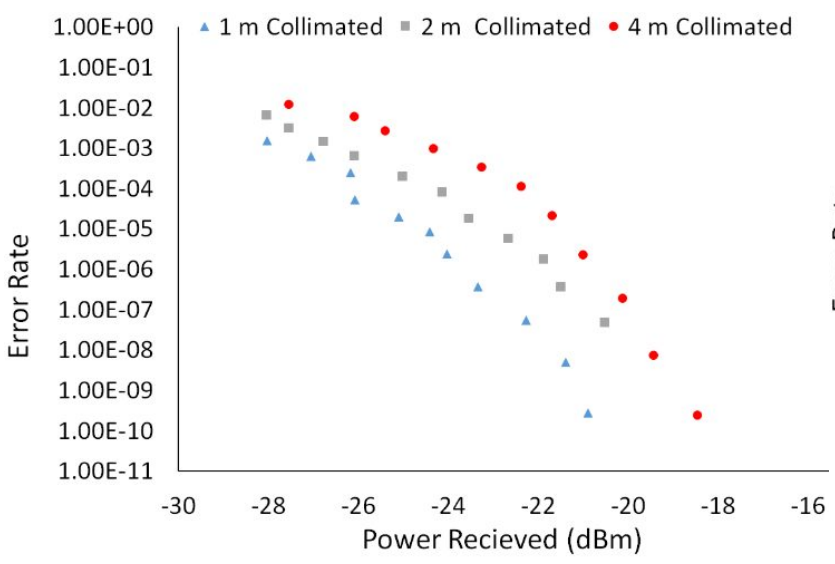

b)

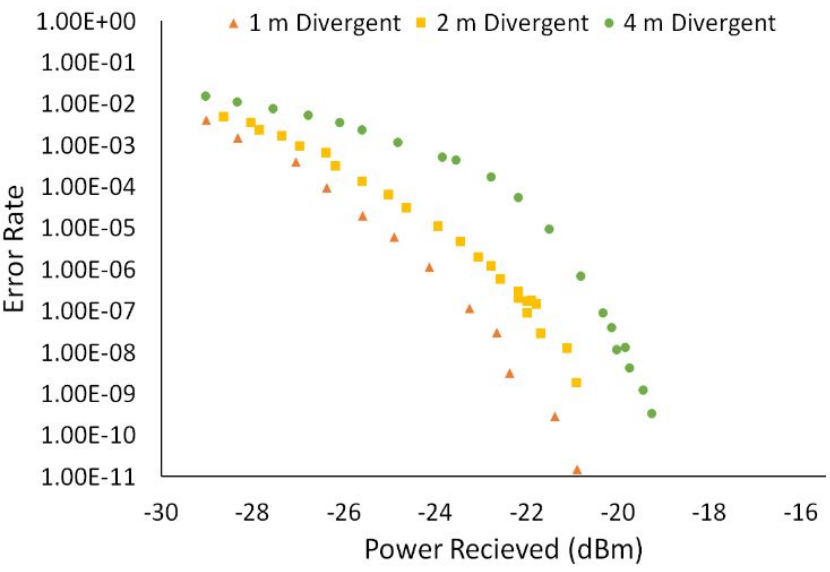

Figure 4. a) BER performance related to $\mathrm{SFP}+$ received power using a collimated beam with various receive multi-mode fiber lengths. b) BER performance related to SFP+ received power using a divergent beam with various receive multi-mode fiber lengths.

In the next data set we compare the free space system to the fiber only system as well as investigate the effect of beam divergence on the free space system. Figure $5 \mathrm{a}$ ) and b) show the BER performance while the cable length is held constant at 1 meter and 4 meters respectively. In both cable lengths the divergent beam FSOL performs the best followed by the collimated beam FSOL then the fiber only system. This result is not as intuitive as above but can also be explained by modal dispersion; more specifically group velocity dispersion ${ }^{6}$ which in some cases is also referred to as differential mode delay $^{6,8}$. Since the fiber only system launches the transmitted beam from a SMF to the MMF, only a few modes of the 
MMF are excited and guided through the cable. In this case, we observe the negative effect of differential mode delay where there is a potential for gaps to occur at the detector between pulses of different modes. These gaps can occur because the pulse width is very short from the high data rate and there is the potential for large delays between modes excited in the fiber. When the delay length between modes approaches the pulse width of the data sequence there becomes the potential for pulse separation at the detector. These gaps or pulse separation at the detector ultimately results in increased bit errors. In the case of the FSOL however many more modes are excited in the MMF. This is because the collected beam spot size from free space is larger than SMF core interface in the fiber only system. Because there are many more modes excited the MMF from the FSOL case, the time delays between modes are more likely to overlap. The result will be pulse broadening and lead to intersymbol interference, but is less likely to cause pulse separation which negatively effects the receiver clock recovery. Therefore the FSOL may help condition the modes in the fiber much the same way a mode conditioning patch cable does in fiber only systems ${ }^{7}$.

Also shown in Figure 5 a) and b), the fiber only system performance degradation is greater at higher signal power levels. This is because the various modes excited in the fiber carry different levels of power. For example, if a particular mode or subset of modes carries most of the total signal power and adjacent modes carry less power, then as the overall power decreases and so will the number of modes contributing to modal dispersion. This will occur when the lower power modes drop below the receiver's threshold as the signal is reduced.

The effect of beam divergence is also shown in Figure 5 a) and b). The divergent beam doesn't form as small of a focus at the fiber as the collimated beam resulting in more modes exited in the MMF. Therefore, the divergence of the beam effects the performance of the system BER. This is evidence by the slight difference in performance between the FSOL using a collimated using a divergent beam. Again this effect is more prominent at higher SNR points due to differential mode delay as discussed above.

a)

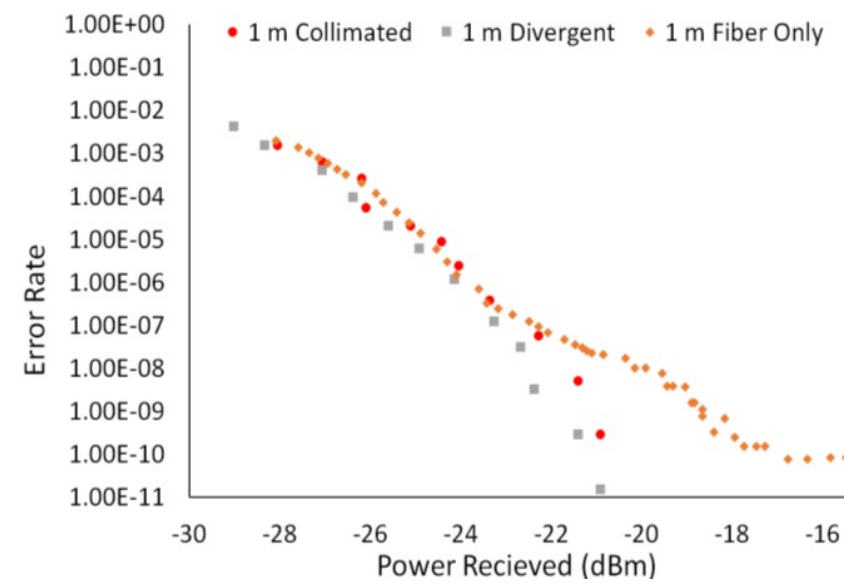

b)

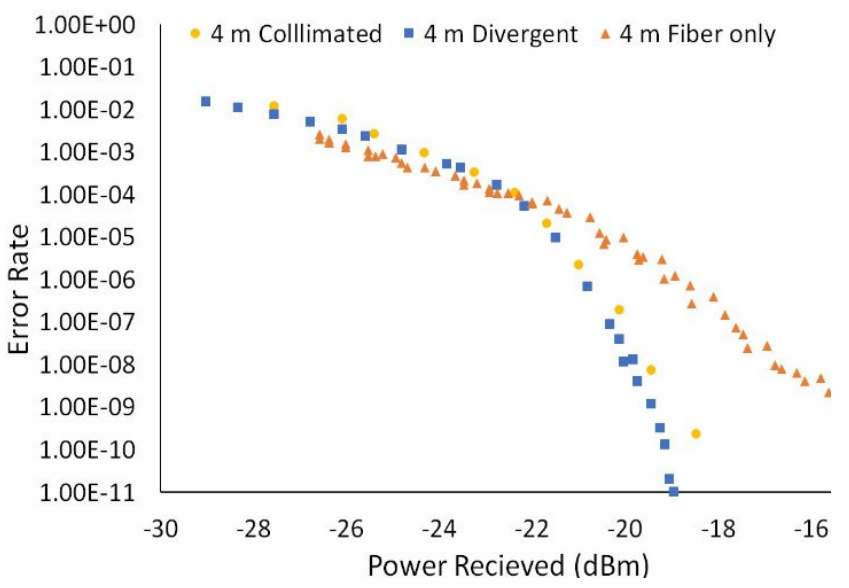

Figure 5. a) BER performance using a 1 meter receive multi-mode fiber. b) BER performance using a 4 meter receive multimode fiber

Figure 6 a) and b) show the effect of system misalignment on BER performance for the 1 meter and 4 meter cable lengths. As expected the shorter cable offers the highest tolerance to misalignment for a given BER due to a lower amount of modal dispersion. Finally, Figure 7 a), b) and c) show the differences in misalignment tolerance relative to BER for both a collimated beam and divergent beam in free space using the 1 and 4 meter cable lengths. For both fiber lengths there exists point at which the collimated beam and divergent beam BER curves cross. In looking back to Figure 2 we notice that the power curves between the collimated beam and divergent beam for each length of cable also cross. This becomes interesting when designing a system for a given misalignment tolerance and expected BER performance. For example from figure 7 a) if a system designer were to use 1e-9 as the required error rate in this system configuration a divergent beam offers a wider misalignment tolerance, however if the metric were more strict or lower than 1e-12 it appears that a collimated beam would be better. This data coupled with the differential mode delay discussion implies that one may be 
able to leverage beam divergence to increase BER performance for specific FSOL's. In order to confirm and quantify this theory a larger data set will be needed consisting of more cases of beam divergence angles.

a)

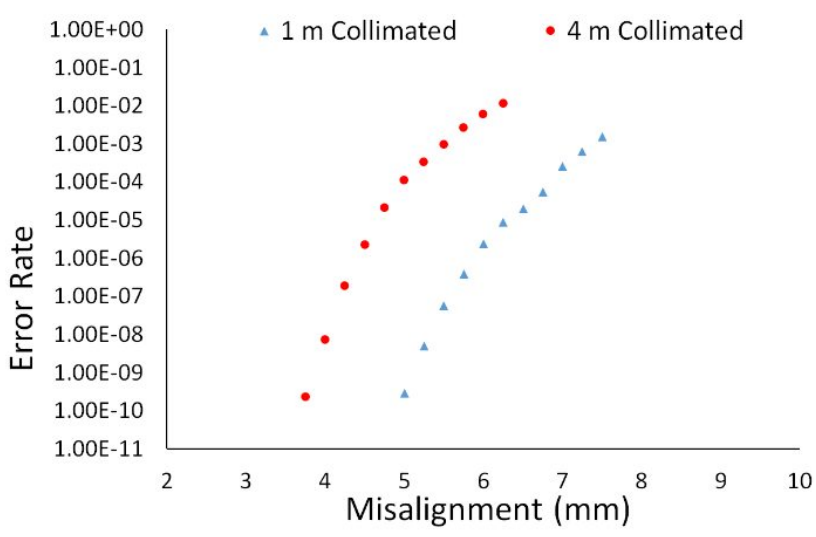

b)

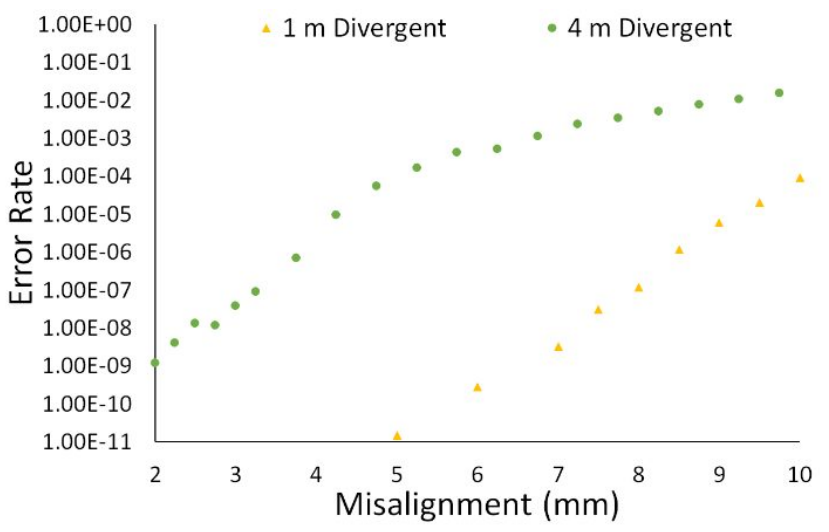

Figure 6. a) BER performance related to lateral misalignment using a collimated beam with various receive multi-mode fiber lengths. b) BER performance related to lateral misalignment using a divergent beam with various receive multi-mode fiber lengths

a)

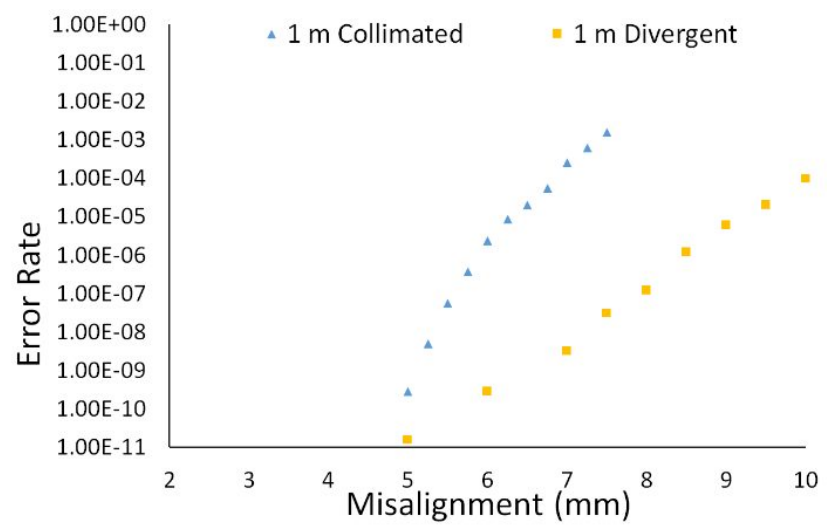

b)

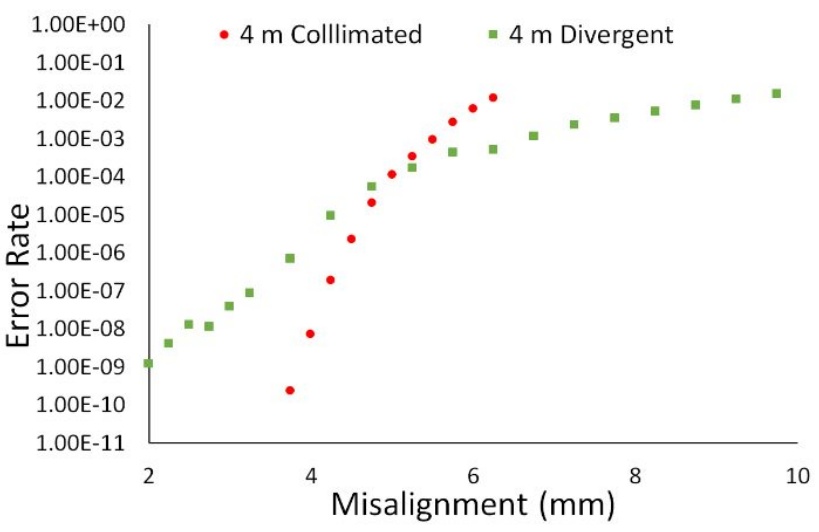

Figure 7. a) BER performance related to lateral misalignment using 1 meter multi-mode fiber. b) BER performance related to lateral misalignment using 4 meter multi-mode fiber.

\section{CONCLUSION}

This paper has presented data for a $10 \mathrm{Gbps}$ FSOL using a $105 \mu \mathrm{m}$ MMF at the receiver. The results show decreased BER performance and misalignment tolerance in the FSOL as the MMF cable length is increased due to modal dispersion. The effect of beam divergence is also presented as the results show a divergent beam has slightly better BER performance compared to a collimated beam with the same SNR. Future work on this system will include a deeper analysis of differential mode delay for both the fiber only and FSOL to better understand the optimal mode excitation in the $105 \mu \mathrm{m}$ fiber to increase BER performance. This can be done by further analyzing the received signal and plotting the eye diagram to determine eye height, eye width and signal jitter for various test configurations. The study will also attempt quantify how beam divergence relates to modal excitation and thus BER performance. 


\section{ACKNOWLEDGEMENTS}

This work is supported by the Advanced Communications and Navigation Division within the NASA Space Communications and Navigation ( $\mathrm{SCaN}$ ) Program. We want to thank Bert Floyd for helping with the system assembly and alignment, Chris Heldman for control system coding, and the SCaN Internship Program (SIP) for giving our students the opportunity to work on this project.

\section{REFERENCES}

[1] Schrage, D., "ISS Truss Flexure Measurement Applying Ka-Band Closed-Loop Tracking", $3^{\text {rd }}$ Annual ISS Research and Development Conference, Chicago, June, 2014

[2] Tedder, S. A., Scheonholz, B. L., and Suddath, S., "Study of Lateral Misalignment Tolerance of a Symmetric Free Space Optical Link for the Development of an Intra ISS Point to Point Wireless Optical Link", 34th AIAA International Communications Satellite Systems Conference, Cleveland, October, 2016.

[3] Guenther, A. H., Pedrotti, L. S., and Roychoudhuri, C., "Optical Waveguides and Fibers," Fundamentals of Photonics, 1st ed., University of Connecticut, Storrs, CT, 2000, pp. 249-292.

[4] Müller, M., Stephens, R., McHugh, R., “Total Jitter Measurement at Low Probability Levels, Using Optimized BERT Scan Method", DesignCon 2005

[5] Ho, K. P., Kahn, J. M., "Statistics of Group Delays in Multimode Fiber with Strong Mode Coupling", Journal of Lightwave Technology, vol. 29, pp. 3119-3128, 2011

[6] Yariv, A., Yeh, P., "Photonics Optical Electronics in Modern Communications" $6^{\text {th }}$ edition, Oxford University Press, NY, 2007

[7] DeCusatis, C. M., "Mode conditioning patch for facilitating signal transmission from single mode optical fiber to multimode optical fiber", US Patent 6415076 B1, issued July 22002

[8] R. Paschotta, article on 'differential mode delay' in the Encyclopedia of Laser Physics and Technology, accessed on 2017-12-02 